Proceedings of the 2012 Winter Simulation Conference

C. Laroque, J. Himmelspach, R. Pasupathy, O. Rose, and A. M. Uhrmacher, eds.

\title{
RARE-EVENT SIMULATIONS FOR EXPONENTIAL INTEGRALS OF SMOOTH GAUSSIAN PROCESSES
}

\author{
Jingchen Liu \\ Gongjun Xu \\ Department of Statistics \\ Columbia University \\ New York, NY, USA
}

\begin{abstract}
In this paper, we consider the rare-event simulation of integrals of exponential functions of smooth Gaussian random processes. In particular, we design importance sampling estimators that are asymptotically efficient. The efficiency analysis consists of the bias control and the variance control relative to the interesting tail probabilities.
\end{abstract}

\section{INTRODUCTION}

Consider a mean zero Gaussian random field $\{f(t): t \in T\}$ with unit variance living on a $d$-dimensional domain $T \subset R^{d}$, that is, for every finite subset of $\left\{t_{1}, \ldots, t_{n}\right\} \subset T,\left(f\left(t_{1}\right), \ldots, f\left(t_{n}\right)\right)$ is a mean zero multivariate Gaussian random vector. Let $\mu(t)$ be a (deterministic) function and $\sigma \in(0, \infty)$ be a positive scalar. Define

$$
I(T) \triangleq \int_{T} e^{\sigma f(t)+\mu(t)} d t .
$$

In this paper, we are interested in efficient simulation for the tail probabilities

$$
w(b)=P\left(I(T)>e^{b}\right)=P\left(\int_{T} e^{\sigma f(t)+\mu(t)} d t>e^{b}\right) .
$$

The current study has its applications in finance, spatial analysis, and many other disciplines (Liu 2012; Liu and Xu 2012b).

The extremes of Gaussian random fields have been intensively studied in literature, with its focus mostly on the development of approximations and bounds for the suprema (Borell 1975; Tsirelson, Ibragimov, and Sudakov 1976; Piterbarg 1996; Sun 1993; Azais and Wschebor 2008; Adler and Taylor 2007). The distribution of the random variable $I(T)$ is studied in the literature when $f(t)$ is a Brownian motion (Yor 1992; Duffie 2001). For general Gaussian random fields, Liu (2012) recently derives the asymptotic approximations of $P\left(\int_{T} e^{\sigma f(t)} d t>b\right)$ as $b \rightarrow \infty$ for three times differentiable and homogeneous Gaussian random fields. Liu and $\mathrm{Xu}$ (2012b) further extends the results to the case when the process $f$ has a varying mean function.

Numerical methods for rare-event analysis of the suprema are studied in Adler, Blanchet, and Liu (2008) and more thoroughly in Adler, Blanchet, and Liu (2012). Simulation study for the integral $I(T)$, on the other hand, is a relatively less developed area. In this paper, we construct an efficient estimator for $w(b)$. As we shall explain in Section 2.2, our approach is based on an importance sampling procedure that mimics the conditional distribution of $f$, given that $\left\{I(T)>e^{b}\right\}$.

The remainder of the paper is organized as follows. In Section 2.1 we introduce some notions of efficiency and computational complexity under the setting of rare-event simulation. Sections 2.2 and 


\section{Liu and $X u$}

2.3 provide the construction of our importance sampling estimator and show the main properties of our algorithm. Some numerical simulations are conducted in Section 3 and detailed proofs of our main theorems are given in Section 4.

\section{MAIN RESULTS}

\subsection{On the Rare-event Simulation}

We first introduce some general notions of rare-event simulations. Given that $w(b)$ converges to zero, for a Monte Carlo estimator $L_{b}$, it is more meaningful to consider the error of $L_{b}$ relative to $w(b)$. This is because a trivial estimator $L_{b}^{*} \equiv 0$ has an error $\left|L_{b}^{*}-w(b)\right|=w(b) \rightarrow 0$. One usually employs the concept of weak efficiency or asymptotic optimality as an efficiency criterion, which is defined as follows.

Definition 1 An estimator $L_{b}$ is said to be weakly efficient or asymptotically optimal in estimating $w(b)$ if $E L_{b}=w(b)$ and

$$
\limsup _{b \rightarrow \infty} \frac{\operatorname{Var}\left(L_{b}\right)}{w^{2-\varepsilon}(b)}=0
$$

for all $\varepsilon>0$.

Asymptotic optimality is a popular efficiency criterion in the rare-event simulation (Asmussen and Glynn 2007). Suppose that one wants to estimate $w(b)$ with certain relative accuracy, that is, to compute an estimator $Z_{b}$ such that for some prescribed $\varepsilon, \delta>0$

$$
P\left(\left|Z_{b} / w(b)-1\right|>\varepsilon\right)<\delta .
$$

Suppose that a weakly efficient estimator of $w(b)$ has been obtained, denoted by $L_{b}$. Let $\left\{L_{b}^{(j)}: j=1, \ldots, n\right\}$ be $n$ i.i.d. copies of $L_{b}$. The averaged estimator

$$
Z_{b}=\frac{1}{n} \sum_{j=1}^{n} L_{b}^{(j)}
$$

has a relative mean squared error equal to $\operatorname{Var}^{1 / 2}\left(L_{b}\right) / n^{1 / 2} w(b)$. A simple application of Chebyshev's inequality yields

$$
P\left(\left|Z_{b} / w(b)-1\right| \geq \varepsilon\right) \leq \frac{\operatorname{Var}\left(L_{b}\right)}{n \varepsilon^{2} w^{2}(b)}
$$

Thus, if $L_{b}$ is an asymptotically optimal (weakly efficient) estimator, it suffices to simulate $n=$ $o\left(\varepsilon^{-2} \delta^{-1} w(b)^{-\varepsilon^{\prime}}\right)$ (for $\varepsilon^{\prime}>0$ ) i.i.d. replicates of $L_{b}$ to achieve the accuracy in (4). Compared with the crude Monte Carlo simulation, which requires $n=O\left(\varepsilon^{-2} \delta^{-1} w(b)^{-1}\right)$, weakly efficient estimators substantially reduce the computational cost.

In addition to the variance control, another issue is that the random fields considered in this paper are continuous objects while computer can only perform discrete simulations. Thus, we must use a discrete object to approximate the continuous fields for the implementation. The bias caused by the discretization must also be well controlled relative to $w(b)$. This will be discussed in Section 2.3.

\subsection{The Change of Measure}

Throughout the discussion, we consider a Gaussian random field $\{f(t): t \in T\}$ living on a domain $T$, which is a $d$-dimensional Borel measurable compact set of $R^{d}$ with piecewise smooth boundary. For $s, t \in T$, we write the covariance function of $f$ as

$$
C(s-t)=\operatorname{Cov}(f(s), f(t))
$$

We shall need the following assumptions: 


\section{Liu and $X u$}

C1 $\quad f$ is homogeneous with $E f(t)=0$ and $E f^{2}(t)=1$ and almost surely at least three times differentiable with respect to $t$.

C2 The Hessian matrix of $C(t)$ at the origin is $-I$, where $I$ is the $d \times d$ identity matrix.

C3 For each $t \in R^{d}$, the function $C(\lambda t)$ is a non-increasing function of $\lambda \in R^{+}$.

C4 The mean function $\mu(t)$ is three-time differentiable. In addition, If $\mu(t)$ is not a constant, the maximum of $\mu(t)$ is not attained at the boundary of $T$.

Condition $\mathrm{C} 1$ is rather a strong assumption. It implies that $C(t)$ is at least 6 times differentiable and the first, third, and fifth derivatives at the origin are all zero. Differentiability is a crucial assumption in this analysis. Condition $\mathrm{C} 2$ is introduced to simplify notations. For any Gaussian process $g(t)$ with covariance function $C_{g}(t)$ and $\Delta C_{g}(0)=-\Sigma$ and $\operatorname{det}(\Sigma)>0, \mathrm{C} 2$ can be obtained by an affine transformation by letting $g(t)=f\left(\Sigma^{1 / 2} t\right)$ and

$$
\int_{T} e^{\mu(t)+\sigma g(t)} d t=\operatorname{det}\left(\Sigma^{-1 / 2}\right) \int_{\left\{s: \Sigma^{-1 / 2} s \in T\right\}} e^{\mu\left(\Sigma^{-1 / 2} s\right)+\sigma f(s)} d s
$$

where for each positive semi-definite matrix $\Sigma$ we let $\Sigma^{1 / 2}$ be a symmetric matrix such that $\Sigma^{1 / 2} \Sigma^{1 / 2}=\Sigma$. Conditions $\mathrm{C} 3$ and $\mathrm{C} 4$ are imposed for technical reasons.

Our algorithm is established using an importance sampling procedure. In this subsection, we propose a change of measure $Q$ on the continuous sample path space and later discretize it for the implementation. This measure is central to our analysis. Let $P$ be the original measure. We describe $Q$ in two ways. First, we specify the simulations of $f$ from $Q$ and then provide its Radon-Nikodym derivative with respect to $P$. Under the measure $Q, f(t)$ is generated according to the following three steps:

1. Simulate a random variable $\tau$ uniformly over $T$ with respect to the Lebesgue measure.

2. Given the realized $\tau$, simulate $f(\tau) \sim N(u-\mu(\tau) / \sigma, 1)$, where, for each $b, u$ satisfies the identity

$$
\left(\frac{2 \pi}{\sigma}\right)^{\frac{d}{2}} u^{-\frac{d}{2}} e^{\sigma u}=e^{b}
$$

3. Given $(\tau, f(\tau))$, simulate the Gaussian process $\{f(t): t \neq \tau\}$ from the original distribution under $P$.

Remark 1 Note that when $b$ is large, equation (5) generally has two solutions. One is on the order of $b / \sigma$; the other one is close to zero. We choose $u$ to be the larger one.

For the measure $Q$ defined above, it is not hard to verify that $P$ and $Q$ are mutually absolutely continuous with the Radon-Nikodym derivative being

$$
\frac{d Q}{d P}=\int_{T} \frac{1}{m e s(T)} \cdot \frac{\exp \left\{-\frac{1}{2}\left(f(t)-u_{t}\right)^{2}\right\}}{\exp \left\{-\frac{1}{2} f(t)^{2}\right\}} d t,
$$

where $\operatorname{mes}(\cdot)$ denotes the Lebesgue measure,

$$
\mu_{t}=u-\mu_{\sigma}(t), \quad \mu_{\sigma}(t)=\mu(t) / \sigma .
$$

The measure $Q$ is constructed such that the behavior of $f$ under $Q$ mimics the behavior of $f$ given the rare event $\left\{I(T)>e^{b}\right\}$ under $P$. In particular, a random variable $\tau$ is first sampled uniformly over $T$, then $f(\tau)$ is simulated with a large mean at level $u-\mu_{\sigma}(\tau)$. This suggests that the high level of the integral $\int_{T} e^{\mu(t)+\sigma f(t)} d t$ is mostly caused by the fact that the field reaches a high level at one location $t^{*}$ that is very close to $\tau$. Therefore, $\tau$ is a random index localizing the maximum of the field. 


\section{Liu and $X u$}

Under the above change of measure, we have the corresponding importance sampling estimator taking the form

$$
L_{b}=\frac{d P}{d Q} 1_{\left(I(T)>e^{b}\right)} .
$$

The second moment of the estimator equals

$$
E^{Q}\left[\left(\frac{d P}{d Q}\right)^{2} ; I(T)>e^{b}\right]=E^{Q}\left[\left(\int_{T} \frac{1}{m e s(T)} \cdot \frac{\exp \left\{-\frac{1}{2}\left(f(t)-u_{t}\right)^{2}\right\}}{\exp \left\{-\frac{1}{2} f(t)^{2}\right\}} d t\right)^{-2} ; I(T)>e^{b}\right]
$$

where we use $E^{Q}$ to denote the expectation under $Q$ and $E$ to denote that under $P$.

\subsection{The Algorithm}

Direct simulation of a continuous random field is typically not a feasible task. Therefore the change of measure proposed in the previous subsection is not directly applicable. To overcome this difficulty, we concentrate on a suitable discretization scheme, still having in mind the change of measure (6).

We create a regular lattice covering $T$ in the following way. Let $G_{N, d}$ be a countable subset of $R^{d}$

$$
G_{N, d}=\left\{\left(\frac{i_{1}}{N}, \frac{i_{2}}{N}, \ldots, \frac{i_{d}}{N}\right): i_{1}, \ldots, i_{d} \in \mathbb{Z}\right\}
$$

That is, $G_{N, d}$ is a regular lattice on $R^{d}$. For each $t=\left(t^{1}, \cdots, t^{d}\right) \in G_{N, d}$, define

$$
T_{N}(t)=\left\{\left(s^{1}, \cdots, s^{d}\right) \in T: s^{j} \in\left(t^{j}-1 / N, t^{j}\right] \text { for } j=1, \cdots, d\right\}
$$

that is the $\frac{1}{N}$-cube intersected with $T$ and cornered at $t$. Furthermore, let

$$
T_{N}=\left\{t \in G_{N, d}: T_{N}(t) \neq \emptyset\right\},
$$

which is the sub-lattice intersecting with $T$. Since $T$ is compact, $T_{N}$ is a finite set. We enumerate the elements in $T_{N}=\left\{t_{1}, \cdots, t_{M}\right\}$, where $M=O\left(N^{d}\right)$. We use

$$
w_{M}(b)=P\left(I_{M}(T)>b\right)
$$

as an approximation of $w(b)$ where

$$
I_{M}(T)=\sum_{i=1}^{M} \operatorname{mes}\left(T_{N}\left(t_{i}\right)\right) \times e^{\sigma f\left(t_{i}\right)+\mu\left(t_{i}\right)} .
$$

We estimate $w_{M}(b)$ by importance sampling, which is based on the change of measure proposed in (6). In particular we define $Q_{M}$ and $P_{M}$ as the discrete versions (on $T_{N}$ ) of $Q$ and $P$ respectively. Then $d Q_{M} / d P_{M}$ takes the form:

$$
\frac{d Q_{M}}{d P_{M}}=\sum_{i=1}^{M} \frac{1}{M} \frac{e^{-\frac{1}{2}\left(f\left(t_{i}\right)-u_{t_{i}}\right)^{2}}}{e^{-\frac{1}{2} f\left(t_{i}\right)^{2}}}=\sum_{i=1}^{M} \frac{1}{M} e^{u_{t_{i}} f\left(t_{i}\right)-\frac{1}{2} u_{t_{i}}^{2}} .
$$

Based on the above change of measure, our algorithm is given as follows:

Algorithm 2 For a given $N$ and the set $T_{N}=\left\{t_{1}, \cdots, t_{M}\right\}$, the algorithm is as follows:

1. Generate $\imath$ uniformly from $\{1, \ldots, M\}$. 


\section{Liu and $X u$}

2. Generate $f\left(t_{l}\right) \sim N\left(u_{t_{l}}, 1\right)$.

3. Given $\left(t_{l}, f\left(t_{l}\right)\right)$, simulate the field $\left(f\left(t_{1}\right), \cdots, f\left(t_{l-1}\right), f\left(t_{l-1}\right), \cdots, f\left(t_{M}\right)\right)$ from the original conditional distribution under $P$.

4. Output

$$
\tilde{L}_{b}=\frac{1_{\left\{I_{M}(T)>e^{b}\right\}}}{\sum_{i=1}^{M} \frac{1}{M} e^{u_{t_{i}} f\left(t_{i}\right)-\frac{1}{2} u_{t_{i}}^{2}}} .
$$

It is not hard to verify that $\tilde{L}_{b}=1_{\left\{I_{M}(T)>e^{b}\right\}} \frac{d P_{M}}{d Q_{M}}$ is an unbiased estimator of $w_{M}(b)$. We have the next theorem to control the bias of the estimator $\tilde{L}_{b}$.

Theorem 3 Suppose $f$ is a Gaussian random field satisfying Conditions C1-C4. For any $\varepsilon_{0}>0$, there exists constant $\kappa_{0}$ such that for any $\varepsilon \in(0,1)$, if $N>\kappa_{0} \varepsilon^{-1-\varepsilon_{0}} u^{2+\varepsilon_{0}}$, then for $b>1$,

$$
\frac{\left|w_{M}(b)-w(b)\right|}{w(b)}<\varepsilon
$$

The next theorem controls the variance of the estimator $\tilde{L}_{b}$.

Theorem 4 Suppose $f$ is a Gaussian random field satisfying Conditions C1-C4. If $N$ is chosen as in Theorem 3, then for any $\varepsilon^{\prime}>0$, we have

$$
\limsup _{b \rightarrow \infty} \frac{E^{Q_{M}} \tilde{L}_{b}^{2}}{w^{2-\varepsilon^{\prime}}(b)}=0 .
$$

We simulate $n$ i.i.d. copies of $\tilde{L}_{b}$ via Algorithm 2, $\left\{\tilde{L}_{b}^{(j)}: j=1, \ldots, n\right\}$, and the averaged estimator is

$$
Z_{b}=\frac{1}{n} \sum_{j=1}^{n} \tilde{L}_{b}^{(j)}
$$

From the discussion in Section 2.1 and Theorems 3 and 4, in order to achieve an $\varepsilon$ relative error with probability at least $1-\delta$, we need to have $n=O\left(\varepsilon^{-2} \delta^{-1} w^{-\varepsilon^{\prime}}(b)\right)$ for any $\varepsilon^{\prime}>0$. Notice that generating a multivariate Gaussian random vector of dimension $M$ is at the most $O\left(M^{3}\right)$ that is caused by computing eigenvectors of the covariance matrix. Thus, the overall computational complexity is $O\left(M^{3}+M^{2} n\right)$.

Remark 5 The algorithms are developed for differentiable fields. It is conceivable that efficient simulation algorithms can be developed for nondifferentiable fields following similar ideas. However, the complexity analysis could be very different mostly due to the size of the discretization. In addition, the asymptotic approximations of $w(b)$ has not yet been developed and it further adds to the difficulty. In this paper, we do not pursue along this generalization.

\section{SIMULATION}

In this section we apply the proposed algorithm to a homogeneous Gaussian random field $\{f(t), t \in[0,1]\}$ with mean zero and covariance function

$$
C(t)=e^{-t^{2}}
$$

We set $\mu(t)=0$ and $\sigma=1$. Then the quantity of interest is

$$
w(b)=P\left(\int_{0}^{1} e^{f(t)} d t>e^{b}\right) .
$$

To implement the algorithm we need to discretize $T$. Here we choose $N=100$ and the detailed simulation is as follows. 


\section{Liu and $X u$}

1. Generate a random variable $\imath \sim$ Uniform $\{1,2, \cdots, 100\}$.

2. Simulate $f\left(\frac{l}{100}\right) \sim N(u, 1)$, where $u$ is calculated from equation (5).

3. Given $l$ and $f\left(\frac{l}{100}\right)$, simulate $\left\{f\left(\frac{i}{100}\right), i=1, \cdots, \imath-1, \imath+1, \cdots, 100\right\}$.

The estimated tail probabilities $w(b)$ along with the estimated standard deviations $s d^{Q}\left(\tilde{L}_{b}\right)=\sqrt{\operatorname{Var}^{Q}\left(\tilde{L}_{b}\right)}$ are shown in Table 1 . All the results are based on $10^{4}$ independent simulations.

Table 1: Estimates of $w(b), s d^{Q}\left(\tilde{L}_{b}\right)$, and $s d^{Q}\left(\tilde{L}_{b}\right) / w(b)$. All results are based on $10^{4}$ independent simulations and thus the standard errors of the estimates are $s d^{Q}\left(\tilde{L}_{b}\right) / 100$.

\begin{tabular}{c|c|c|c} 
& Est. & $s d^{Q}\left(\tilde{L}_{b}\right)$ & $s d^{Q}\left(\tilde{L}_{b}\right) /$ Est. \\
\hline$b=3$ & $8.62 \mathrm{e}-04$ & $2.10 \mathrm{e}-03$ & 2.43 \\
$b=5$ & $7.23 \mathrm{e}-08$ & $2.32 \mathrm{e}-07$ & 3.20 \\
$b=7$ & $8.56 \mathrm{e}-14$ & $3.51 \mathrm{e}-13$ & 4.10 \\
$b=9$ & $1.42 \mathrm{e}-21$ & $7.69 \mathrm{e}-21$ & 5.40
\end{tabular}

\section{PROOF OF THEOREMS 3 AND 4}

Before proceeding to the proofs of Theorems 3 and 4, we first introduce a few useful lemmas. The first one is known as the Borel-TIS inequality, which was proved independently by Borell (1975), Tsirelson, Ibragimov, and Sudakov (1976).

Lemma 6 (Borel-TIS) Let $f(t), t \in \mathscr{U}, \mathscr{U}$ is a parameter set, be mean zero Gaussian random field. $f$ is almost surely bounded on $\mathscr{U}$. Then,

$$
E\left(\sup _{\mathscr{U}} f(t)\right)<\infty
$$

and

$$
P\left(\max _{t \in \mathscr{U}} f(t)-E\left[\max _{t \in \mathscr{U}} f(t)\right] \geq b\right) \leq e^{-\frac{b^{2}}{2 \sigma_{\mathscr{U}}^{2}}}
$$

where

$$
\sigma_{\mathscr{U}}^{2}=\max _{t \in \mathscr{U}} \operatorname{Var}[f(t)]
$$

The following lemma provides an asymptotic approximation for tail probability $w(b)$ (see Theorem 3.4 in Liu and $\mathrm{Xu}(2012 \mathrm{~b})$ ).

Lemma 7 Consider a Gaussian random field $\{f(t): t \in T\}$ living on a domain $T$ satisfying conditions C1-C4. Then,

$$
w(b)=(1+o(1)) \int_{T} G(t) \cdot u^{d-1} \exp \left\{-\frac{\left(u-\mu_{\sigma}(t)\right)^{2}}{2}\right\} d t
$$

where $u$ is as defined in (5), $\mu_{\sigma}(t)=\mu(t) / \sigma, G(t)$ is defined as

$$
G_{0} \cdot \exp \left\{\frac{d \cdot \mu(t)+2|\partial \mu(t)|^{2}+\sum_{i=1}^{d} \partial_{i i}^{2} \mu(t)}{2 \sigma^{2}}\right\}
$$

and $G_{0}$ is some constant only depending on $\sigma$ and the covariance function of $f$.

The next lemma provides an asymptotic approximation of the density function of $\log I(T)$ (Liu and Xu 2012a). 


\section{Liu and $X u$}

Lemma 8 Suppose that conditions C1-C4 are satisfied. Let $F^{\prime}(x)$ be the probability density function of $\log I(T)=\log \int_{T} e^{\sigma f(t)+\mu(t)} d t$. Then the following approximation holds as $x \rightarrow \infty$

$$
F^{\prime}(x)=(1+o(1)) \sigma^{-2} x \cdot w(x) .
$$

With the above preparations, we are ready to prove Theorems 3 and 4 .

\subsection{Proof of Theorem 3}

For some $A>0$, let

$$
\mathscr{L}=\left\{\sup _{t \in T}|\partial f(t)| \leq A\left(1-u^{-2} \log \varepsilon\right) u\right\}
$$

Then by Lemma 6, we can choose $A$ sufficiently large (independent of $u$ and $\varepsilon$ ) and have that

$$
P\left(\mathscr{L}^{c}\right)=P\left(\sup _{t \in T}|\partial f(t)|>A\left(1-u^{-2} \log \varepsilon\right) u\right)=o(1) \varepsilon w(b) .
$$

Therefore,

$$
\begin{aligned}
\left|w_{M}(b)-w(b)\right| & =\left|P\left(I_{M}(T)>e^{b}, \mathscr{L}\right)-P\left(I(T)>e^{b}, \mathscr{L}\right)\right|+o(1) \varepsilon w(b) \\
& =\left|P\left(I_{M}(T)>e^{b}, I(T)<e^{b}, \mathscr{L}\right)+P\left(I_{M}(T)<e^{b}, I(T)>e^{b}, \mathscr{L}\right)\right|+o(1) \varepsilon w(b) .
\end{aligned}
$$

On the set $\mathscr{L}$, we have that there exists a positive constant $c_{1}$ such that

$$
\begin{aligned}
\left|I_{M}(T)-I(T)\right| & =\left|\sum_{i=1}^{M} \operatorname{mes}\left(T_{N}\left(t_{i}\right)\right) \times e^{\sigma f\left(t_{i}\right)+\mu\left(t_{i}\right)}-\sum_{i=1}^{M} \int_{T_{N}\left(t_{i}\right)} e^{\sigma f(t)+\mu(t)} d t\right| \\
& \leq c_{1} \min \left\{I(T), I_{M}(T)\right\} \cdot \sup _{t \in T}|\partial f(t)| / N,
\end{aligned}
$$

which implies that

$$
\begin{aligned}
(12) & \leq P\left(e^{b}\left(1-c_{1} \frac{A\left(1-u^{-2} \log \varepsilon\right) u}{N}\right)<I(T)<e^{b}\left(1+c_{1} \frac{A\left(1-u^{-2} \log \varepsilon\right) u}{N}\right)\right)+o(1) \varepsilon w(b) \\
& \leq O(1) \frac{\left(1-u^{-2} \log \varepsilon\right) u}{N} \times b \times w(b)+o(1) \varepsilon w(b) .
\end{aligned}
$$

The last step of the above equation is due to the results of Lemma 8. Note that $\sigma u / b \rightarrow 1$. Thus it is sufficient to choose $N=O\left(\varepsilon^{-1-\varepsilon_{0}} u^{2+\varepsilon_{0}}\right)$ so that

$$
\text { (12) } \leq \varepsilon \cdot w(b),
$$

which completes our proof. 


\section{Liu and $X u$}

\subsection{Proof of Theorem 4}

Consider the change of measure in (9),

$$
\frac{d Q_{M}}{d P_{M}}=\sum_{i=1}^{M} \frac{1}{M} \frac{e^{-\frac{1}{2}\left(f\left(t_{i}\right)-u_{t_{i}}\right)^{2}}}{e^{-\frac{1}{2} f\left(t_{i}\right)^{2}}}=\sum_{i=1}^{M} \frac{1}{M} e^{-\frac{1}{2} u_{t_{i}}^{2}+f\left(t_{i}\right) u_{t_{i}}}
$$

For the $t_{i}$ 's in the interior of $T$, we have that $\operatorname{mes}\left(T_{N}\left(t_{i}\right)\right) \sim \operatorname{mes}(T) / M$; for those that are close to the boundary, we have that $\operatorname{mes}\left(T_{N}\left(t_{i}\right)\right)<\operatorname{mes}(T) / M$. Thus, we have

$$
\begin{aligned}
& E^{Q_{M}}\left[\tilde{L}_{b}^{2}\right]=E^{Q_{M}}\left[\frac{1}{\left(\sum_{i=1}^{M} \frac{1}{M} e^{-\frac{1}{2} u_{t_{i}}^{2}+f\left(t_{i}\right) u_{t_{i}}}\right)^{2}} ; \sum_{i=1}^{M} \operatorname{mes}\left(T_{N}\left(t_{i}\right)\right) \times e^{\sigma f\left(t_{i}\right)+\mu\left(t_{i}\right)}>e^{b}\right] \\
& \leq E^{Q_{M}}\left[\frac{1}{\left(\sum_{i=1}^{M} \frac{1}{M} e^{\left.-\frac{1}{2} u_{t_{i}}^{2}+f\left(t_{i}\right) u_{t_{i}}\right)^{2}}\right.} ; 2 \times \frac{\operatorname{mes}(T)}{M} \sum_{i=1}^{M} e^{\sigma f\left(t_{i}\right)+\mu\left(t_{i}\right)}>e^{b}\right] \\
& \leq e^{\left(u-\min _{t} \mu_{\sigma}(t)\right)^{2}} \cdot E^{Q_{M}}\left[\frac{1}{\left(\frac{1}{M} \sum_{i=1}^{M} e^{u_{i} f\left(t_{i}\right)}\right)^{2}} ; 2 \times \frac{\operatorname{mes}(T)}{M} \sum_{i=1}^{M} e^{\sigma f\left(t_{i}\right)+\mu\left(t_{i}\right)}>e^{b}\right] .
\end{aligned}
$$

Note that for $b$ large enough

$$
\frac{1}{M} \sum_{i=1}^{M} e^{u_{t_{i}} f\left(t_{i}\right)}=\frac{1}{M} \sum_{i=1}^{M} e^{u_{t_{i}}\left(f\left(t_{i}\right)+\mu_{\sigma}\left(t_{i}\right)\right)-u_{t_{i}} \mu_{\sigma}\left(t_{i}\right)} .
$$

There are two types of $t_{i}$ 's: $f\left(t_{i}\right)+\mu_{\sigma}\left(t_{i}\right)>0$ and $f\left(t_{i}\right)+\mu_{\sigma}\left(t_{i}\right)<0$. Furthermore, notice that

$$
u_{t_{i}} \mu_{\sigma}\left(t_{i}\right) \leq u \max _{t \in T} \mu_{\sigma}(t)-\min _{t \in T} \mu_{\sigma}^{2}(t)
$$

we have that

$$
\begin{aligned}
& \frac{1}{M} \sum_{i=1}^{M} e^{u_{t_{i}} f\left(t_{i}\right)} \geq e^{-u \max _{t \in T} \mu_{\sigma}(t)+\min _{t \in T} \mu_{\sigma}^{2}(t)} \times \frac{1}{M} \sum_{i=1}^{M} e^{u_{t_{i}}\left(f\left(t_{i}\right)+\mu_{\sigma}\left(t_{i}\right)\right)} \\
& \geq e^{-u \max _{t \in T} \mu_{\sigma}(t)+\min _{t \in T} \mu_{\sigma}^{2}(t)} \\
& \times \frac{1}{M}\left[\sum_{\left\{f\left(t_{i}\right)+\mu_{\sigma}\left(t_{i}\right) \geq 0\right\}} e^{\left(u-\max _{t \in T} \mu_{\sigma}\left(t_{i}\right)\right)\left(f\left(t_{i}\right)+\mu_{\sigma}\left(t_{i}\right)\right)}\right. \\
& \left.+\sum_{\left\{f\left(t_{i}\right)+\mu_{\sigma}\left(t_{i}\right)<0\right\}} e^{\left(u-\min _{t \in T} \mu_{\sigma}\left(t_{i}\right)\right)\left(f\left(t_{i}\right)+\mu_{\sigma}\left(t_{i}\right)\right)}\right] \\
& \geq \delta_{0} e^{-u \max _{t \in T} \mu_{\sigma}(t)} \times\left[\frac{1}{M} \sum_{i=1}^{M} e^{\left(u-\max _{t \in T} \mu_{\sigma}(t)\right)\left(f\left(t_{i}\right)+\mu_{\sigma}\left(t_{i}\right)\right)}\right],
\end{aligned}
$$

where $\delta_{0} \in(0,1)$ is some positive constant chosen to be small. Then by Jensen's inequality, on the set

$$
2 \times \frac{\operatorname{mes}(T)}{M} \sum_{i=1}^{M} e^{\sigma f\left(t_{i}\right)+\mu\left(t_{i}\right)}>e^{b}
$$


we have that

$$
\left(\frac{1}{M} \sum_{i=1}^{M} e^{u_{i} f\left(t_{i}\right)}\right)^{2} \geq \delta_{0}^{2} e^{-2 u \max _{t \in T} \mu_{\sigma}(t)} \cdot\left(\frac{e^{b}}{2 \times m e s(T)}\right)^{2\left(u-\max _{t \in T} \mu_{\sigma}(t)\right) / \sigma}
$$

Therefore, there exists a constant $c_{1}$ such that

$$
\begin{aligned}
(13) & \leq e^{\left(u-\min _{t} \mu_{\sigma}(t)\right)^{2}} \cdot \delta_{0}^{-2} e^{2 u \max _{t \in T} \mu_{\sigma}(t)} \cdot\left(\frac{e^{b}}{2 \times \operatorname{mes}(T)}\right)^{-2\left(u-\max _{t \in T} \mu_{\sigma}(t)\right) / \sigma} \\
& \leq e^{-u^{2}+c_{1} u \log u} .
\end{aligned}
$$

The last step is due to the fact that $b / u \rightarrow \sigma$. Now combining the above result with the approximation of $w(b)$ in Lemma 7 , we have that $-2 \log (w(b)) / u^{2} \rightarrow 1$ and thus for any $\varepsilon^{\prime}>0$

$$
\limsup _{b \rightarrow \infty} \frac{E^{Q} \tilde{L}_{b}^{2}}{w(b)^{2-\varepsilon^{\prime}}}=0
$$

which completes our proof.

\section{ACKNOWLEDGEMENT}

This research is supported in part by Institute of Education Sciences, through Grant R305D100017, NSF CMMI-1069064, and NSF SES-1123698.

\section{REFERENCES}

Adler, R., J. Blanchet, and J. Liu. 2008, December. "Efficient Simulation for Tial Probabilities of Gaussian Random Fields". In Proceedings of the 2008 Winter Simulation Conference, edited by S. J. Mason, R. R. Hill, L. Moench, O. Rose, T. Jefferson, and J. W. Fowler, 328-336. Piscataway, New Jersey: Institute of Electrical and Electronics Engineers, Inc.

Adler, R., J. Blanchet, and J. Liu. 2012. "Efficient Monte Carlo for Large Excursions of Gaussian Random Fields". Annals of Applied Probability 22 (3): 1167-1214.

Adler, R., and J. Taylor. 2007. Random fields and geometry. Springer.

Asmussen, S., and P. Glynn. 2007. Stochastic Simulation: Algorithms and Analysis. New York, NY, USA: Springer.

Azais, J. M., and M. Wschebor. 2008. "A general expression for the distribution of the maximum of a Gaussian field and the approximation of the tail". Stochastic Processes and Their Applications 118 (7): $1190-1218$.

Borell, C. 1975. "The Brunn-Minkowski inequality in Gauss space". Inventiones Mathematicae 30 (2): 207-216.

Duffie, D. 2001. Dynamic asset pricing theory. 3rd ed. Princeton, N.J.: Princeton University Press.

Liu, J. 2012. "Tail Approximations of Integrals of Gaussian Random Fields". Annals of Probability 40 (3): $1069-1104$.

Liu, J., and G. Xu. 2012a. "On the Density Functions of Integrals of Gaussian Random Fields". Journal of/Advances in Applied Probaiblity to appear:http://stat.columbia.edu/ jcliu/paper/grf10.pdf.

Liu, J., and G. Xu. 2012b. "Some Asymptotic Results of Gaussian Random Fields with Varying Mean Functions and the Associated Processes". Annals of Statistics 40:262-293.

Piterbarg, V. I. 1996. Asymptotic methods in the theory of Gaussian processes and fields. Providence, R.I.: American Mathematical Society.

Sun, J. Y. 1993. "Tail probabilities of the maxima of gaussian random-fields". Annals of Probability 21 (1): 34-71. 


\section{Liu and $X u$}

Tsirelson, B., I. Ibragimov, and V. Sudakov. 1976. "Norms of Gaussian sample functions". Proceedings of the Third Japan-USSR Symposium on Probability Theory (Tashkent, 1975) 550:20-41.

Yor, M. 1992. "On Some Exponential Functionals of Brownian-Motion". Advances in Applied Probability 24 (3): 509-531.

\section{AUTHOR BIOGRAPHIES}

JINGCHEN LIU is an Assistant Professor in the Department of Statistics at Columbia University. Jingchen received his Ph.D. in statistics from Harvard University in June, 2008. His email address is jcliu@stat.columbia.edu.

GONGJUN XU is a Ph.D. student in the Department of Statistics at Columbia University. His email address is gongjun@stat.columbia.edu. 